\title{
EP-167
}

\section{Total laparoscopic versus robotic-assisted laparoscopic pancreaticoduodenectomy; which one is better?}

\author{
Munseok CHOI', Ho Kyoung HWANG ${ }^{2,3}$, Woo Jung LEE ${ }^{2,3}$, Chang Moo KANG ${ }^{* 2,3}$ \\ 'Department of Surgery, Yongin Severance Hospital, Yonsei University College of Medicine, Yongin, Korea \\ ${ }^{2}$ Division of Hepatobiliary and Pancreatic Surgery, Department of Surgery, Yonsei University College of Medicine, Seoul, Korea \\ ${ }^{3}$ Pancreaticobiliary Cancer Clinic, Yonsei Cancer Center, Severance Hospital, Seoul, Korea
}

Introduction: Minimally invasive pancreaticoduodenectomy is a difficult and complex operation. Since the development of the robotic platform, the number of reports on robotic-assisted pancreatic surgery has been on the rise. This study aimed to compare the technical feasibility and safety between the LPD and the robotic-assisted laparoscopic pancreaticoduodenectomy (RALPD).

Methods: From September 2012 to August 2020, this study enrolled 257 patients who underwent minimally invasive pancreaticoduodenectomy for periampullary tumor. Of these patients, 207 underwent the LPD, and 50 underwent RALPD. We performed a 1:1 propensity score-matched (PSM) analysis.

Results: There was no difference in demographics, including the size of the pancreatic duct, pancreatic texture, and vascular resection. Operation time and estimated blood loss were similar, as was the incidence of complications, such as POPF, delayed gastric emptying, and postoperative hemorrhage. When comparing LPD and RALPD in only patients with a pancreatic duct size of $2 \mathrm{~mm}$ or less, there was no difference between the two groups in short-term surgical outcomes, including clinically relevant POPF (CR-POPF). In multivariable analysis, robotic reconstruction was not a predictive factor for CR-POPF, and the only soft pancreatic texture was a predictive factor for CR-POPF (HR 3.887, 95\% confidence interval 1.121 to $13.480, p=0.032$ ).

Conclusions: RALPD and LPD are one of the approaches for safe and effective PD, and they are technically equivalent regardless of the size of the pancreatic duct. Considering the high cost of robotic surgery, surgeons need to prepare not only robotic but also the pure laparoscopic technique for PD to fulfill the goal of MIS. 